\title{
Reduced mismatch negativity and increased variability of brain activity in schizophrenia
}

\author{
Todor Jordanov*, Tzvetan Popov, Nathan Weisz, Thomas Elbert, Isabella Paul-Jordanov, Brigitte Rockstroh \\ Department of Psychology, University of Konstanz, Konstanz, Germany
}

\section{A R T I C L E I N F O}

\section{Keywords:}

MEG

Schizophrenia

Mismatch negativity

Noise

Signal variability

Trial-by-trial variability

\section{H I G H L I G H T S}

- Perceptual and memory functions in schizophrenia (reflected by a reduced MMN) are unrelated to increased amplitude variability of the brain response.

- Reduced stimulus-related phase-locking and deficient auditory sensory memory may characterize the disorder.

- Without considering the contribution of diminished temporal stability of neuronal network dynamics to schizophrenia the impaired performance of brain function compared to healthy controls cannot be reliably modeled.

\section{A B S T R A C T}

Objectives: Schizophrenia patients commonly exhibit smaller amplitudes of mismatch negativity (MMN) than in controls. It remains unclear whether this results from deficient processes indexed by MMN or 'normally' though more variable processing. The present magnetoencephalographic study addressed this question by analyzing intra-individual trial-by-trial variability and MMN amplitude.

Methods: Twenty inpatients meeting ICD criteria for schizophrenia and 18 healthy controls participated in an auditory oddball experiment. The neuromagnetic mismatch field (MMNm) was defined as the difference waveform deviant minus standard tone response. Variability index (VI) in different frequency bands was quantified as trial-by-trial variation of stimulus-evoked responses and epoch-by-epoch variation of signal amplitude during a resting condition.

Results: Patients displayed a smaller MMNm amplitude and higher VI during the oddball experiment and during the resting condition than in controls. VI and MMNm amplitude were correlated in controls, but not in patients.

Conclusion: Reduced MMN in schizophrenia cannot be explained by augmented variability of brain activity; deficient auditory sensory memory and stimulus related phase-locking may characterize the disorder.

Significance: Understanding the contribution of diminished temporal stability of neuronal network dynamics to schizophrenia is crucial in modeling the impact of such instability on performance and thus for understanding deviant attention and memory functions.

\section{Introduction}

Event-related brain potentials (ERP) have been used to understand perceptual or cognitive abnormalities in schizophrenia patients relative to healthy controls. Smaller mean amplitudes in patients than in controls have been confirmed for various ERP

\footnotetext{
* Corresponding author. Address: Department of Psychology, University of Konstanz, PO Box 25, D-78457 Konstanz, Germany. Tel.: +49 75318830 86; fax: +497531884601.
}

E-mail address: todor:iordanov@uni-konstanz.de (T. Jordanov). components and for difference waveforms in oddball designs, in which rare deviant events are embedded in a stream of repeated standard events (Callaway et al., 1970; Winterer et al., 2000 2004; Garrido et al., 2009; Rosburg et al., 2004; Näätänen, 1992; Näätänen et al., 2007; Umbricht and Krljes, 2005). Different hypotheses have related the smaller ERP amplitudes to deviant or deficient perceptual or cognitive processing in schizophrenia, while the extent to which smaller mean amplitudes result from less activity as a function of less efficient processing or from less activity as a function of more variable but otherwise unimpaired processing across trials is still discussed. Augmented response 
variability across trials has been demonstrated for amplitude and latency of ERP components such as N100, P300 and P50 gating ratio in schizophrenia patients (Roth et al., 2007; lyer and Zouridakis, 2008; Jansen et al., 2010). If, as suggested by computational models (Rolls et al., 2008), this temporal variability reflects less orchestrated neuronal firing or reduced signal-to-noise ratio, discussing the functional meaning of reduced MMN as an index of deficiency has to be challenged. It thus seems crucial to specify the contribution of trial-by-trial ERP variability to the mean evoked response.

The present study addressed the inter-trial variability of cortical processing for the auditory mismatch negativity (MMN), a reliable response to physically deviant events embedded in a stream of repeated stimuli (Näätänen et al., 2007; Tervaniemi et al., 2005). The MMN is reduced in amplitude in schizophrenia (reviews by Rosburg et al., 2004; Michie, 2001; Näätänen and Kähkönen, 2009) and is considered to indicate of perceptual accuracy, sensory learning and memory (Umbricht and Krljes, 2005; Garrido et al., $2008,2009)$. Hence, reduced MMN or smaller mismatch fields than the electromagnetic analog of the MMN (MMNm, Kreitschmann-Andermahr et al., 1999; Pekkonen et al., 2002; Kircher et al., 2004) in schizophrenia patients are discussed as signs of reduced integrity in these perceptual and memory functions of the auditory cortex (Michie, 2001; Pekkonen et al., 2002; Magno et al., 2008; Näätänen and Kähkönen, 2009; Turetsky and Moberg, 2009).

Various designs containing the violation of an acoustic regularity elicit MMN and MMNm. Amplitudes vary according to design features (e.g., deviation in frequency, duration, intensity, stimulus onset asynchrony, oddball ratio, rhythms or musical sequences; Näätänen et al., 2004; Thönnessen et al., 2008; Matsubayashi et al., 2008; Tervaniemi et al., 2005; Deouell and Bentin, 1998; Garrido et al., 2009), but also according to gender and personality traits (Matsubayashi et al., 2008; Tervaniemi et al., 2005). Reduced MMN amplitudes in schizophrenia patients are more reliably found for duration than for frequency deviants (Rosburg et al., 2004); they vary according to illness duration and severity (Näätänen and Kähkönen, 2009; Kirino, 2007; Umbricht and Krljes, 2005; Todd et al., 2008) but seem robust against medication effects (Michie, 2001). Temporal variability of the MMN across trials has not been scrutinized, although Roth et al. (2007) reported increased latency and amplitude variability of the P300 component in schizophrenia patients. Augmented temporal variability of neuronal network activity in schizophrenia might be deduced from computational neuroscience models of reduced stability of attractor networks and, as a consequence, reduced signal-to-noise ratio of brain activity (Rolls et al., 2008).

The present study seeks to add further evidence of such instability and its relationship to mean ERP amplitude by analyzing trial-by-trial variability in the MMN paradigm. Verifying the contribution of instability of processing to mean amplitudes is crucial for understanding mechanisms indexed by reduced MMN such as deficient detection accuracy or auditory memory. In addition, epoch-by-epoch variation of brain activity was analyzed for a resting condition in order to test the hypothesis of a general (relative to mainly event-related) augmented temporal variability of cortical network dynamics (Rolls et al., 2008). Both variability indices (VI) were tested for relationships with the MMNm amplitude. We hypothesize that: (1) a negative correlation between MMNm and VI measured in the oddball design indicates that MMNm could result from deficient and/or from inconsistent processing; (2) a negative correlation between $\mathrm{MMNm}$ and epoch-by-epoch variability (VI) during resting state indicates that deficient auditory stimulus processing is related to the temporal variability of brain activity in general; and (3) uncorrelated measures indicate that deficient processing and adequate though variable processing might act additively or interactively in the generation of auditory
Table 1

Demographic characteristics for the group of schizophrenia patients and the group of healthy controls for measurement conditions (oddball design and resting state).

\begin{tabular}{llll}
\hline & Patients & Controls & Inferential tests \\
\hline $\begin{array}{l}\text { Oddball design } \\
N\end{array}$ & 20 & 18 & \\
Age $(M \pm \mathrm{SD})$ & $31 \pm 8.5$ & $27.7 \pm 4.8$ & $F(1,36)=2, p>.1$ \\
Gender: females/males & $1 / 19$ & $7 / 11$ & $\mathrm{Chi}^{2}(1)=6.55, p<.05$ \\
Years of education $(M \pm \mathrm{SD})$ & $12 \pm 2.1$ & $17.4 \pm 2$ & $F(1,36)=66.8, p=0$ \\
Handedness: left/right & $4 / 16$ & $2 / 16$ & $\mathrm{Chi}^{2}(1)=0.6, p>.1$ \\
Resting state & & & \\
$N$ & 16 & 14 & \\
Age $(M \pm \mathrm{SD})$ & $30.3 \pm 7.3$ & $30.4 \pm 7.1$ & $F(1,28)<1, p>.1$ \\
Gender: females/males & $1 / 15$ & $4 / 10$ & $\mathrm{Chi}$ \\
Years of education $(M \pm \mathrm{SD})$ & $12.1 \pm 2.1$ & $18 \pm 2$ & $F(1,28)=6.7, p>.1$ \\
Handedness: left/right & $3 / 13$ & $2 / 12$ & $\mathrm{Chi}^{2}(1)<1, p>.1$ \\
\hline
\end{tabular}

Note: $M \pm \mathrm{SD}:$ mean \pm standard deviation.

perceptual and/or memory performance such as induced in oddball designs. MEG seems advantageous for these analyses as: (a) MEG measurements are less affected by conductivities of the skull and the scalp than EEG measurements so that interpretation of MEG signals does not require preliminary knowledge of the thicknesses and conductivities of the tissues in the head (Hämäläinen et al., $1993)$; (b) the magnetic field drops rapidly with distance $\left(1 / \mathrm{r}^{2}\right)$, which improves the separation of simultaneous activities in the left and in the right brain hemispheres (Reite et al., 1999).

\section{Methods}

\subsection{Subjects}

Twenty inpatients meeting the ICD-10 criteria for paranoid-hallucinatory schizophrenia (F20.0) and 18 psychiatrically healthy controls participated in the oddball experiment (see Table 1 for demographic characteristics). Resting state data were available for 16 patients and 14 controls (see Table 1). Patients and controls did not differ with respect to age, but controls had more years of education.

Patients were diagnosed by experienced senior psychiatrists using ICD-10 criteria. The psychiatrist or psychologist responsible for the particular patient also assessed symptom severity with the Brief Psychiatric Rating Scale (BPRS; Lukoff et al., 1986), Beck Depression Inventory (BDI-II; Beck et al., 1996) and Global Assessment of Functioning Scale (GAF; DSM-IV-TR; American Psychiatric Association, 2000; see Table 2). These assessments were made on the day of the MEG measurement. All patients were on psychoactive medication (see Table 2). Healthy controls were included if they did not meet criteria for a lifetime diagnosis of mental illness (screened with the MINI interview; Ackenheil et al., 1999) and

Table 2

Clinical characteristics of schizophrenia patients.

\begin{tabular}{lll}
\hline Characteristic & $M \pm S D$ & Range \\
\hline BPRS & $47.3 \pm 9.0$ & $26-65$ \\
GAF & $34.1 \pm 8.3$ & $20-60$ \\
BDI & $12.5 \pm 11.2$ & $1-48$ \\
Number of hospitalizations & $5.5 \pm 6$ & $1-23$ \\
Medication: CPZ & $843 \pm 583$ & $245-2700$ \\
Number of patients on: & & \\
Typical neuroleptics & 2 & \\
Atypical neuroleptics & 13 & \\
Both & 5 & \\
\hline
\end{tabular}

Note: BPRS: Brief Psychiatric Rating Scale, BPRS (Lukoff et al., 1986); GAF: Global Assessment of Functioning Scale; DSM-IV-TR (American Psychiatric Association, 2000); BDl: Beck Depression Inventory (Beck et al., 1996); CPZ: cllorpromazine equivalents. $M \pm S D$ : mean \pm standard deviation. 
were free of psychoactive medication. For all subjects, exclusion criteria included any history of head trauma with loss of consciousness. All subjects were informed about the measurement procedures and gave their written consent prior to measurements.

\subsection{Stimuli and design}

The study design was approved by the Ethics Committee of the University of Konstanz. Within an auditory oddball design including a random sequence of 2200 stimuli $80 \%$ (1800) were standard tones ( $500 \mathrm{~Hz}, 20 \mathrm{~ms}$ duration), $10 \%$ (200) were frequency-deviant tones $(550 \mathrm{~Hz}, 20 \mathrm{~ms}$ duration) and $10 \%(200)$ duration-deviant tones $\left(500 \mathrm{~Hz}, 60 \mathrm{~ms}\right.$ duration). ${ }^{1}$ Stimuli were presented binaurally with a $270 \pm 15$-ms offset-to-onset interval. A minimum of three and a maximum of six standards were presented between two deviants. Tones were delivered via plastic tubes to the subject within the shielded MEG recording chamber and presented at $50 \mathrm{~dB}$ above the subjective hearing level, which was determined separately for each ear. No task was involved, but participants were asked to keep their eyes focused on a small fixation point throughout the measurement.

\subsection{Data acquisition and analysis}

MEG was recorded using a 148-channel magnetometer (MAGNESTM 2500 WH, 4D Neuroimaging, San Diego, USA) while participants were in a lying position. Data were continuously recorded with a sampling rate of $678.17 \mathrm{~Hz}$ and a real bandpass filter of $0.1-200 \mathrm{~Hz}$. For artifact control, the vertical and horizontal electro-oculogram (EOG) was recorded using four electrodes placed near the left and right temporal canthus and above and below the right eye using a SynAmps amplifier (NEUROSCAN Laboratories, Sterling, VA, USA). The subject's nasion, left and right ear canal, and head shape were digitized prior to measurement with a Polhemus 3Space ${ }^{\circledast}$ Fasttrack.

Global noise was filtered from the MEG data offline by subtracting non-biological external noise that was recorded by 11 MEG reference sensors. Prior to subtraction, reference channels were multiplied with individually calculated fixed weight factors. This noise reduction procedure has little or no influence on biological signals as the distance between the reference sensors and the subject's head is large $(M \pm S D 25.8 \pm 6.0 \mathrm{~cm}$, min $15.5 \mathrm{~cm}$, max $36.5 \mathrm{~cm}$ ) relative to the distance between the head and adjacent sensors.

MEG data were analyzed using the Matlab-based FieldTrip toolbox developed at the Donders Institute for Brain, Cognition and Behavior (http://fieldtrip.fcdonders.nl/). Data segments containing eye blinks, muscle artifacts or superconducting quantum interference device (SQUID) jumps were rejected using an artifact rejection function. For each trial, the variance across all sampling points was calculated separately for each channel. Whenever the maximum value across channels exceeded a threshold value of $1 \times 10^{-24} \mathrm{fT}^{2}$, the trial was classified as artifact-contaminated

\footnotetext{
1 The present study was part of a larger project in which patients were assigned to an auditory discrimination training including frequency changes. Accuracy of frequency discrimination was therefore of particular interest in the pre-training assessment, part of which constituted the present MMN analysis. Duration deviants were nevertheless included, with the aim of assessing general or specific auditory processing deficits. Both stimulus conditions had already been combined within single studies, for instance, by Deacon et al. (1998), Deouell and Bentin (1998), Michie et al. (2000), Petermann et al. (2009), Magno et al. (2008), Horton et al. (2010), Fisher et al. (2011), albeit with different aims than those of the present study. These studies showed independent differential effects of both dimensions or similar effects for duration and frequency deviants if the deviation was large enough. Although MMN as a measure of discrimination accuracy of changes in frequency was of particular interest the MMNm and VI for duration deviants was analyzed as well.
}

and excluded from further analysis. The threshold value was empirically determined by computing the variance for random samples of trials with and without artifacts. Variance of artifactfree trials did not exceed $1 \times 10^{-24} \mathrm{fT}^{2}$, whereas the variance on trials with eye movement artifacts ranged from $0.5 \times 10^{-24}$ to $6.4 \times 10^{-24} \mathrm{fT}^{2}$. Analyses of averaged responses were based on planar gradients of the MEG field distribution determined using the nearest-neighbor method (Bastiaansen and Knösche, 2000). The horizontal and vertical components of the estimated planar gradients approximate the signal measured by MEG systems with planar gradiometers (see Fig. 1). As the maximal activity of planar gradients is typically located above the source, this analysis can be used to estimate loci of activity sources (Hämäläinen et al., 1993).

Continuous data were filtered using a $1-\mathrm{Hz}$ high-pass zerophase as well as a 45- $\mathrm{Hz}$ low-pass zero-phase Butterworth filter of second order. For MMNm analysis, epochs of $300 \mathrm{~ms}$ duration following stimulus onset relative to a 100 -ms prestimulus interval were determined separately for standards and deviants. Resting state data were segmented into 1 -s epochs, resulting in an average of 250 epochs. The same number of trials per subject were chosen for further analyses by selecting the first 1000 artifact-free responses to standards for each subject, the first 100 artifact-free responses to each frequency duration deviant as well as the first 150 artifact-free resting-state epochs.

The neuromagnetic mismatch field (MMNm) was calculated by subtracting the magnetic fields per sensor evoked by standards from those evoked by frequency deviants and the duration deviants separately.

Variability in the oddball design was defined as trial-by-trial variation of stimulus-evoked responses (Möcks et al., 1988) calculated for each MEG sensor, each time point and each stimulus type (standards and deviants). The VI (variability index) estimate is the mean magnitude of the difference between every single trial (or epoch in the resting data) and the average across trials. VI is calculated as a function of time over the entire trial/epoch length,

$V I(t)=\sqrt{\frac{1}{N-1} \Sigma_{i=1}^{N}\left(x_{i}(t)-\bar{x}(t)\right)^{2}}$

with $N$ representing the number of trials/epochs, $t$ indicating the current time point, $x_{i}(t)$ the value of the $i$-th trial/epoch in the time point $t$ and $\bar{x}(t)$ the average over all trials or epochs in the time point $t$. Results are reported as VI in femto-Tesla (fT), the square root of the noise power $\left(\mathrm{fT}^{2}\right) . \mathrm{VI}(t)$ in both oddball and resting state data was also estimated for frequency bands after having band-pass filtered the data: delta-theta $(1-7 \mathrm{~Hz})$, alpha $(8-12 \mathrm{~Hz})$, beta $(13-24 \mathrm{~Hz})$ and gamma $(25-45 \mathrm{~Hz})$. The mean MMNm amplitudes, the $\mathrm{VI}(t)$ for oddball, and the $\mathrm{VI}(t)$ for resting state were first averaged over time (100-240 ms for MMNm; $0-250 \mathrm{~ms}$ for $\mathrm{VI}(t)$ in the oddball design; $0-250 \mathrm{~ms}$ for the $\mathrm{VI}(t)$ during resting state) and then compared between groups (schizophrenia patients versus controls). Comparisons were corrected for family-wise error rate using a non-parametric $t$-test-based randomization test (Maris and Oostenveld, 2007). This procedure effectively controls for multiple comparisons and allows for the identification of sensor clusters of significant group differences. A cluster was defined as a set of adjacent sensors (defined as sensors at less than $3.2 \mathrm{~cm}$ distance, yielding on average three neighbors per sensor) that exhibited similar differences between groups in $t$-value and magnitude. Group differences were considered statistically robust for a sensor cluster when the significance level exceeded 95\%. Signals of significant sensor clusters were averaged and subjected to a one-way analysis of variance (ANOVA) for group comparison. Since VI has been reported to be lower in females than in males (Winterer et al., 2004), separate ANOVAs were calculated: (i) for the entire sample, comparing patients and controls with the 
$\mathrm{HC}$

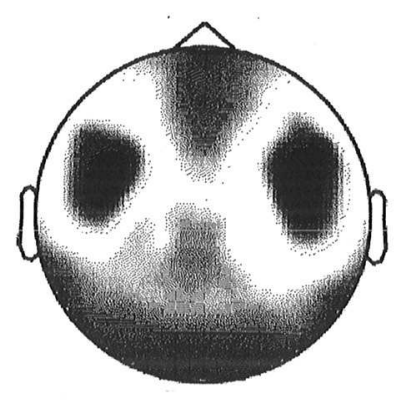

SZ

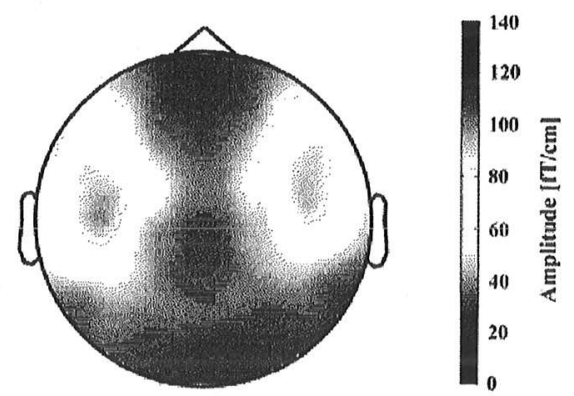

Fig. 1. Grand average mismatch fields averaged separately for healthy controls (left) and schizophrenia patients (right) for axial gradiometers transformed into planar gradiometers. The color bar indicates field strength in femto Tesla $/ \mathrm{cm}(\mathrm{fT} / \mathrm{cm})$. (For interpretation of the references to color in this figure legend, the reader is referred to the
web version of this article.)

between-subject factor 'Group'; (ii) for the control group, comparing male and female subjects with the between-subject factor 'Gender'; and (iii) for the male sample with the between-subject factor 'Group', comparing male patients and male controls. The relationship between average event-related responses (MMNm) and VI values was probed by Pearson correlation coefficients. Since the number of subjects in the different groups was quite small regarding statistical power, the size of all statistical effects was calculated. Hedges's $g$ (Hedges and Olkin, 1985) was used as a somewhat more accurate version of Cohen's $d$ as it adds a correction factor for small samples.

\section{Results}

As illustrated in Figs. 1 and 2, MMNm in the time interval 100$240 \mathrm{~ms}$ after stimulus onset was smaller in schizophrenia patients than in controls for both frequency and duration deviants. MMNm did not differ between frequency and duration deviants, as indicated by a repeated measures ANOVA with the between factor Group and within factor Deviant, which did not confirm a significant interaction Group $\times$ Deviant $(F(1,36)=1.05, p>.1)$ or main effect Deviant $(F(1,36)=0.35, p>.5)$, but only a significant main effect $\operatorname{Group}(F(1,36)=8.1, p<.01)$. Therefore, results are reported for the amplitude averaged across MMNm types. The group difference was confirmed when only male subjects were considered $(F(1,28)=11.7, p<.01$; see also Table 3$)$. As evident in Fig. 2 and confirmed by a randomization cluster, statistics differences were prominent at bilateral fronto-temporal sensors.

In the oddball design, VI was significantly larger in patients than in controls $(F(1,36)=15.44, p<.01)$. As evident in Fig. 3, differences were not confined to stimulus onset but evident across the entire time interval for responses to standards and deviants. The
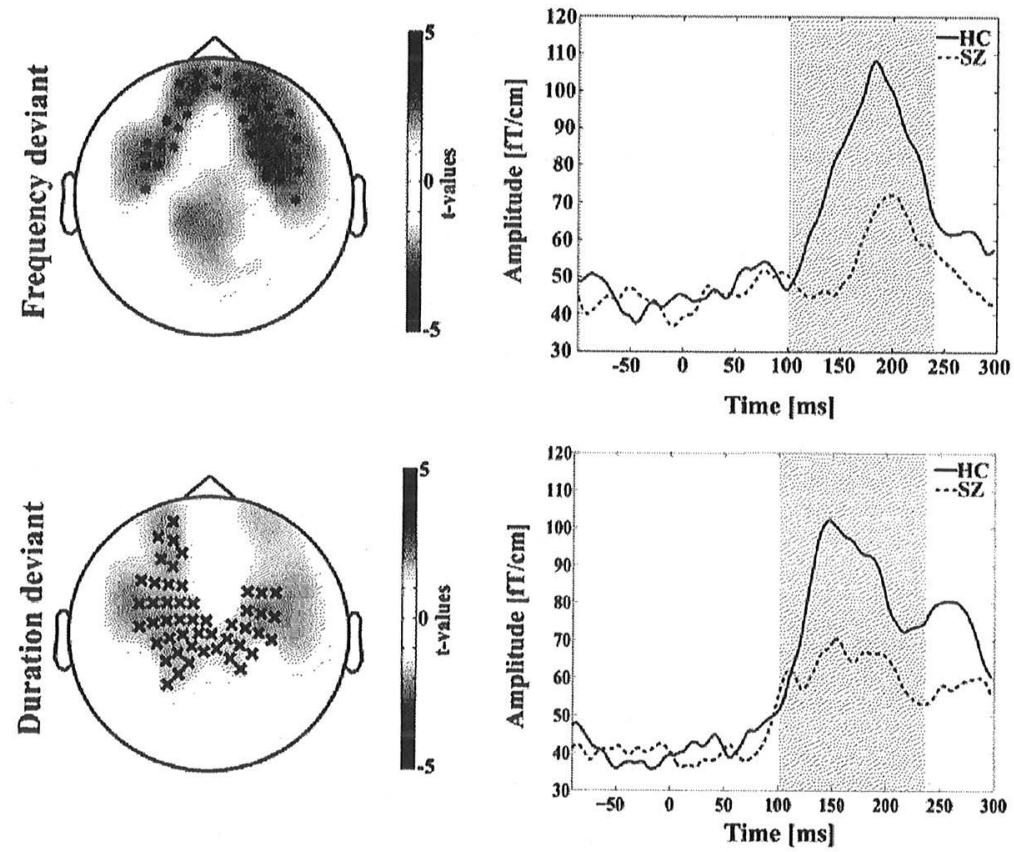

Fig. 2. Left: Scalp distribution of MMNm group differences in the time window 100-240 ms after stimulus, onset for both types of deviants (frequency and duration). The range of $t$-values is represented by color shading (color bar indicates $t$-values). Sensors contributing to a cluster of significant group difference $(p<.025)$ are represented by asterisks and of significant group difference with $p<.05$ with " $X$ ". Right: The quadratic mean of the mismatch response across significant sensors is plotted over time ( 100 to $300 \mathrm{~ms}$ ) for healthy controls (solid line) and schizophrenia patients (dashed line). The MMNm can be seen as a prominent deflection between 100 and $240 \mathrm{~ms}$ after tone onset. (For interpretation of the references to color in this figure legend, the reader is referred to the web version of this article.) 
Table 3

Group differences in MMNm and VI.

\begin{tabular}{|c|c|c|c|c|}
\hline Scores & Statistical effect & $\begin{array}{l}\text { A: } \\
\text { Comparison patients - controls } \\
\text { all subjects }\end{array}$ & $\begin{array}{l}\text { B: } \\
\text { Comparison patients - controls } \\
\text { male subjects only }\end{array}$ & $\begin{array}{l}\text { C: } \\
\text { Comparison female - } \\
\text { male controls only }\end{array}$ \\
\hline MMNm planar gradient $(\mathrm{fT} / \mathrm{cm})$ & $\begin{array}{l}\text { Sample size } \\
\text { Group } \\
\text { Effect size }\end{array}$ & $\begin{array}{l}20 \text { vs. } 18 \\
F(1,36)=8.1, p<.01 \\
g=-0.96\end{array}$ & $\begin{array}{l}19 \text { vs. } 11 \\
F(1,28)=11.7, p<.01 \\
g=-1.33\end{array}$ & $\begin{array}{l}7 \text { vs. } 11 \\
F(1,16)=2.2, p>.1 \\
g=-0.63\end{array}$ \\
\hline Variability index oddball design (fT) & $\begin{array}{l}\text { Sample size } \\
\text { Group } \\
\text { Effect size }\end{array}$ & $\begin{array}{l}20 \text { vs. } 18 \\
F(1,36)=15.44, p<.01 \\
g=1.26\end{array}$ & $\begin{array}{l}19 \text { vs. } 11 \\
F(1,28)=5.08, p<.05 \\
g=0.83\end{array}$ & $\begin{array}{l}7 \text { vs. } 11 \\
F(1,16)=3.21, p<.1 \\
g=-0.82\end{array}$ \\
\hline Variability index resting state (fT) & $\begin{array}{l}\text { Sample size } \\
\text { Group } \\
\text { Effect size }\end{array}$ & $\begin{array}{l}16 \text { vs. } 14 \\
F(1,28)=13.51, p<.01 \\
g=1.31\end{array}$ & $\begin{array}{l}15 \text { vs. } 10 \\
F(1,23)=7.49, p<.05 \\
g=1.08\end{array}$ & $\begin{array}{l}4 \text { vs. } 10 \\
F(1,12)=0.64, p>.1 \\
g=-0.44\end{array}$ \\
\hline
\end{tabular}

Note: Dependent variables MMNm (planar gradient in $\mathrm{fT} / \mathrm{cm}$ ), VI in oddball design and VI in the resting state were compared: (A) between all patients and controls, $(B)$ for male patients and controls, (C) for male and female controls. Group differences were expressed as ANOVA main effect for group (A, B) or gender (C) or as effect size according
to Hedge's g.

less variable VI time course for standards may be explained by the larger number of 1000 averages relative to 100 averages selected for each type of deviant. Differences were significant at bilateral temporo-frontal sensor clusters. As in the MMNm group, differences remained when considering male subjects only $(F(1,28)=5.08, p<.05)$.

VI determined from resting state MEG (Fig. 4) was significantly more pronounced in schizophrenia patients than in controls $(F(1,28)=13.51, p<.01$; for male subjects, $F(1,23)=7.49, p<.05)$. Group differences were mainly confined to left temporo-frontal sensor clusters. No effect of gender was found in the control group $(F(1,12)<1)$. VI determined for the different frequency bands (Fig. 5) showed significant group differences of resting state VIs in the delta-theta band and mainly in a left temporal sensor cluster $(p<.05)$. In contrast to this, VI in the oddball design was significantly larger in patients than in controls for all except the alpha band (delta-theta: bilateral temporal sensor cluster, $p<.05$; beta: bilateral temporal and frontal sensor cluster, $p<.05$; gamma: bilateral temporal and posterior sensor cluster, $p<.05$ ).

Correlation analysis verified positive relationships between MMNm amplitude and VI (in delta-theta frequency band) in the oddball design in healthy controls $(r=.59, p=.01)$ but not in patients $(r=-.08$, n.s. $)$. A test for homogeneity of the regression slopes confirmed the significant difference between groups $(F=9.73, p<.01, g=-1.04)$ (Fig. 6). A positive correlation $(r=.63$, $p<.01)$ confirmed that trial-by-trial variability in the oddball design and epoch-by-epoch variability in the resting condition were related to each other.

Neither MMNm nor VI varied with clinical characteristics in patients, as indicated by the correlation coefficient for symptom
S
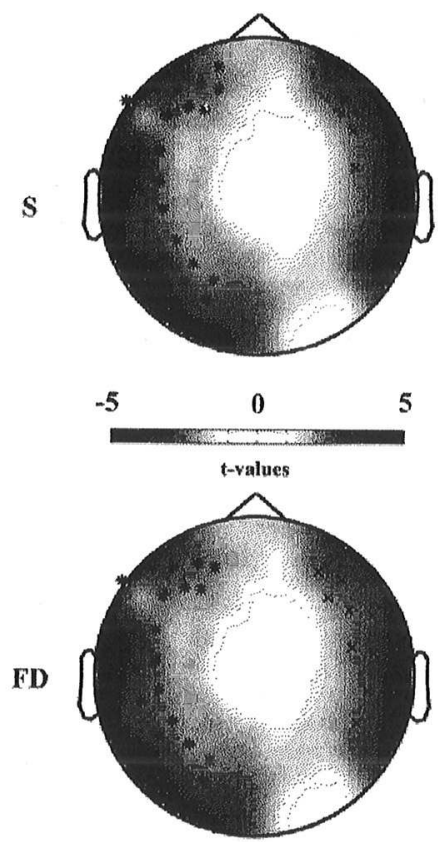
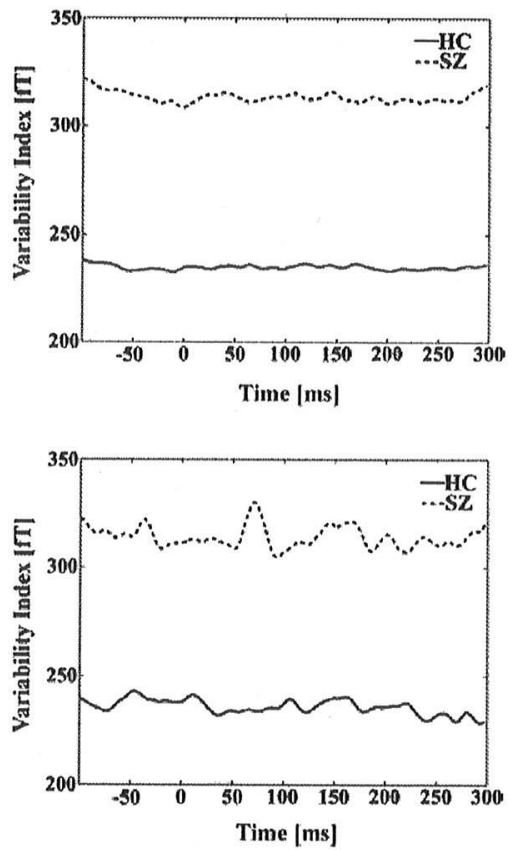

Fig. 3. Left: Scalp distribution of group differences of variability indices (VI) following standard (S, top row) and frequency deviant (FD, bottom row) stimuli. The range of $t$-values is represented by color shading and the color bar indicates $t$-values. Sensors contributing to a cluster of significant group difference are marked by asterisks (for $p<.025$ ) or $\mathrm{X}$ (for $p<.05$ ). Right: The quadratic mean of $\mathrm{VI}(t)$ across significant sensors is plotted for patients (dotted lines) and controls (solid lines), as well as for standard tones (top), and deviant tones (bottom) for the time interval -100 to $300 \mathrm{~ms}$. (For interpretation of the references to color in this figure legend, the reader is referred to the web version of this article.) 

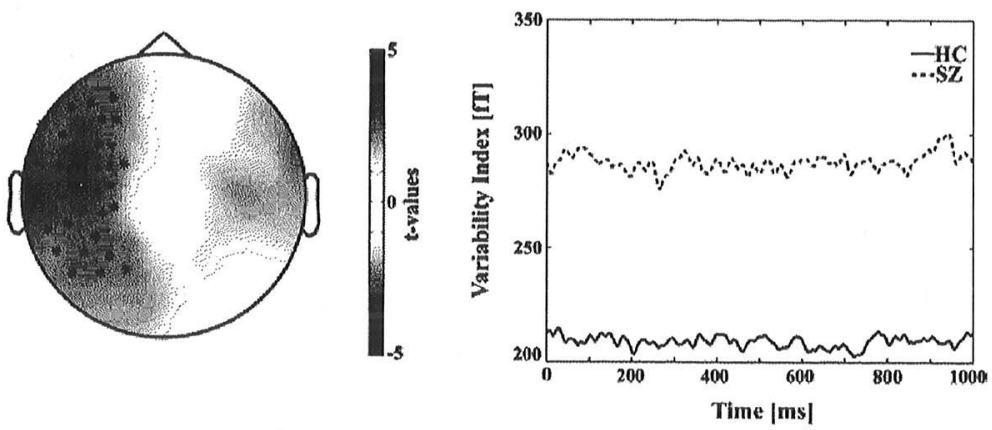

Fig. 4. Left: Scalp distribution of group differences of variability indices (VI) during resting state. The range of $t$-values is represented by color shading and the color ba indicates $t$-values. Sensors contributing to a cluster of significant group difference are marked by asterisks $(p<.05)$. Right: The quadratic mean of $V l(t)$ across significant sensors is plotted for patients (dotted lines) and controls (solid lines) for a $1 \mathrm{~s}$ time interval. (For interpretation of the references to color in this figure legend, the reader is referred to the web version of this article.)

severity (BPRS, MMNm: $r=-.002$, VI: $r=.18$.), global functioning (GAF, MMNm: $r=.3$, VI: $r=-.22$,) and depression (BDI, MMNm: $r=-.07$, VI: $r=-.21$ all $p>.1$ ). Similarly, MMNm and VI did not vary with medication indexed by chlorpromazine equivalents (MMN, $r=.15, \mathrm{VI}, r=-.05, p>.2$ ).

\section{Discussion}

Previous results had suggested that an increased trial-by-trial variability contributes to smaller ERP components in schizophrenia patients (Roth et al., 2007). The present study confirmed differences in $\mathrm{MMNm}$ and in variability indices (VI) between groups, whereas relationships between $\mathrm{VI}$ and averaged MMNm amplitude in patients were not found.

MMNm was smaller in patients than in controls, regardless of the type of stimulus deviance (frequency or duration). This is in line with reports of reduced MMN in schizophrenia evident in different designs (Thönnessen et al., 2008; Michie et al., 2000; Horton et al., 2010; Pakarinen et al., 2010) and suggests that reduced MMN is a robust phenomenon (Michie, 2001). These reports discussed the meaning of reduced MMN in schizophrenia as indexing deficient automatic detection of irregularities in auditory stimulus sequence and auditory memory trace formation. However, this discussion should consider alternative explanations for small mean amplitude. The extent to which temporal instability of processing (reflected by trial-by-trial variability of MMNm) constitutes a source is discussed below.

MMNm in patients did not vary with type of deviant, medication, symptom severity or global level of functioning. Although reduced MMN in schizophrenia patients has been found in designs using frequency and duration deviants, differences in the robustness and intensity of the effect between the types of deviants have also been reported (Rosburg et al., 2004). In the present study, it cannot be ruled out that the combination of the two deviants within the same tone sequence may have reduced a differential effect of each deviant type on MMNm along with generally smaller MMNm. Fisher et al. (2011) concluded from a similar result that increased deviant probability impairs the development of separate deviant memory traces. Whereas robustness against medication effects is in line with previous reports (Michie, 2001), variation with symptom measures and duration of illness has also been frequently reported (Näätänen and Kähkönen, 2009; Kirino, 2007; Umbricht and Krljes, 2005; Todd et al., 2008). However, further conclusions from the present study are limited, as only general symptom severity and not more specific symptom profiles could be considered. Reports on relationships between MMN and positive or negative symptoms are not consistent (Rosburg et al., 2004), and significant relationships may vary with larger and less heterogeneous samples. Moreover, as the number of hospitalizations does not adequately reflect the duration of an illness, present results cannot be compared to results reported by other groups. If the impact of
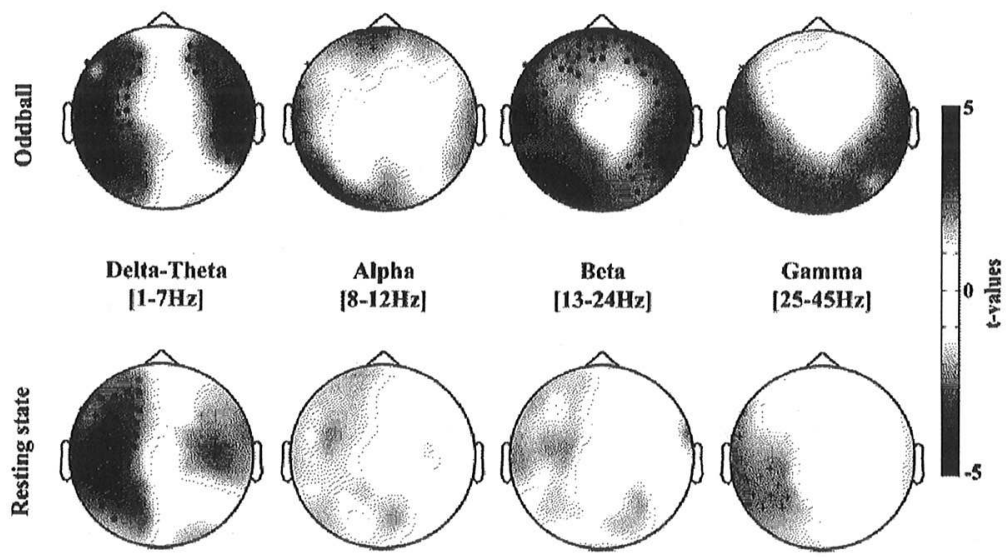

Beta

$[13-24 H z]$ $[25-45 \mathrm{~Hz}]$
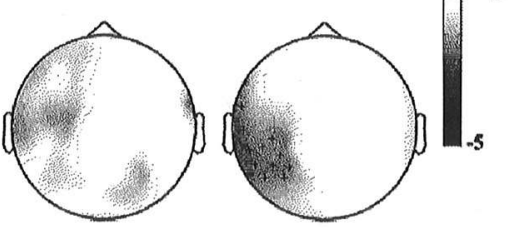

Fig. 5. VI magnitude differences between patients and controls in frequency bands of interest. Sensor clusters that distinguish groups are marked with asterisks (for $p<.025$ ) or X (for $p<.05$ ); sensor clusters that distinguish groups only as a trend $(p<.1)$ are marked by ' + '. The color bar indicates the range of $t$-values. (For interpretation of the references to color in this figure legend, the reader is referred to the web version of this article.) 


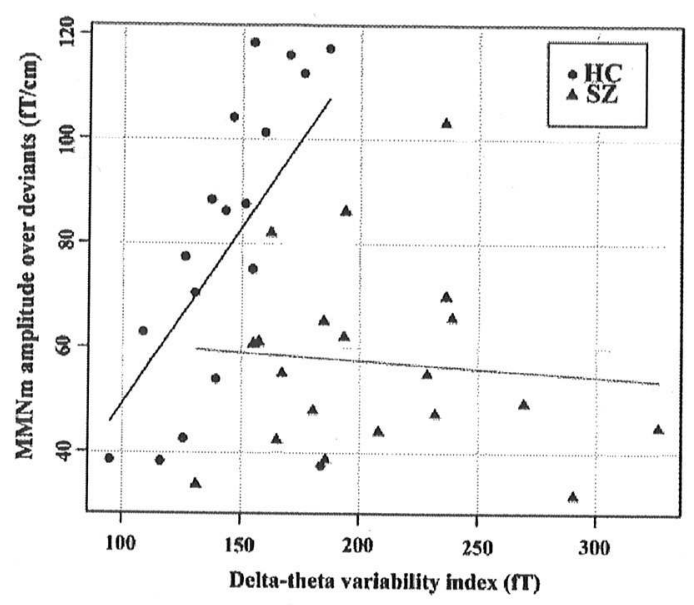

Fig. 6. Relationship between V1 magnitude in the delta-theta frequency band and MMNm amplitude obtained in the oddball paradigm are displayed for individual subjects: open triangles indicate schizophrenia patients; open circles indicate controls. The regression homogeneity test yielded significant difference in the slopes $(p<.01)$.

clinical and design characteristics on MMNm and its trial-by-trial variability is a target in further studies, sample size and selection should be tuned to this goal.

Analysis of trial-by-trial variability of MMN and epochby-epoch variability under resting conditions confirmed larger variability in schizophrenia patients than in controls. Increased signal variability has been reported for schizophrenia (Winterer et al. $2000,2004,2006$ ), suggesting reduced stability of attractor networks and, as a consequence, reduced signal-to-noise ratio of activity as derived from computation neuroscience models (Rolls et al., 2008). Orchestrated non-stimulus-related firing of an attractor network can strengthen its functionality (Elbert et al., 1994) and stability of attractor networks is essential for forming and maintaining sensory memory (Rolls et al., 2008). The present result of augmented VI may index this diminished stability and thereby point to a dysfunction that may become evident in deficient processing.

Prominent VI in schizophrenia patients could be the consequence of reduced phase-locking, greater amplitude variability or a combination of both. The VI measure used in this study is not suitable for distinguishing between these sources of variability. However, the finding of augmented VI under oddball and resting conditions suggests at least partial contribution of background activity to VI. Similarly, Winterer et al. (2000) did not find a relationship between variability and ERP amplitude using a measure comparable to the present VI. Additional separate analyses of amplitude variability and phase locking, however, suggested that increased VI in the delta-theta range resulted from a decrease in phase locking.

Group differences in VI varied with frequency band and measurement conditions: schizophrenia patients displayed higher VI than healthy controls in lower frequency bands under resting as well as oddball conditions. Furthermore, augmented slow wave activity in schizophrenia patients has already been reported in other studies (Fehr et al., 2001, 2003; Rockstroh et al., 2007; Siekmeier and Stufflebeam, 2010) and might provide a basis for more pronounced temporal variability and thus higher VI.

VI of higher-frequency bands only differed between groups during the oddball design. Oscillatory activity in the beta/gamma range has been linked to attention-triggering task engagement and stimulus encoding (e.g., Yabe et al., 2005; Thönnessen et al., 2008; Tallon-Baudry et al., 1999). Reduced gamma band activity in schizophrenia has been found in arithmetic and spatial tasks (e.g., Kissler et al., 2000) and auditory reaction tasks (e.g., Leicht et al., 2010; Popov et al., 2011); the augmented VI of higher frequency bands in the present patient sample may therefore reflect less stable engagement of distributed cortical networks in initial stimulus encoding. Auditory stimulation activates the temporal lobes and this activation manifests itself in more synchronized oscillatory activity in higher frequency bands in healthy controls. In schizophrenia patients, more variable network activity may contribute to higher signal variability in higher frequency bands alongside lower stimulus-evoked (synchronized) delta-theta band response.

Group differences of VI were prominent at fronto-temporal sensor clusters during auditory stimulation and at left-temporal sensor clusters during resting state. Augmented VI has been reported for frontal sensors in schizophrenia patients in EEG studies (Winterer et al., 2000, 2004), and diminished network stability in schizophrenia has been modeled mainly for prefrontal cortex networks (Rolls et al., 2008). The slight incongruity between the present and previous studies could be explained by the more radial orientation of frontal sources, as MEG sensors are mainly sensitive to fields generated by tangential sources (Garrido et al., 2009). Rinne et al. (2000) and Pekkonen et al. (2002) found temporal sources in an oddball experiment with both MEG and EEG, whereas prefrontal sources were only present in EEG. A more likely explanation might be that the topography of auditory sources is characterized by a widespread fronto-central negativity in EEG but has a temporal distribution in MEG.

Present results confirmed positive correlation coefficients for healthy controls and no relationship for patients. Both results seem counter-intuitive at first glance. For healthy controls, we may assume that peripheral stimuli generate ERPs by reorganizing the phase spectra of existing ongoing activity and by increasing event-related signal power (Winterer et al., 2000). Both processes may have occurred simultaneously in controls, as suggested by the positive correlation of resting-state and stimulus-related VI. In this regard, both processes may have contributed to VI and hence to a positive correlation of VI and MMNm amplitude. Reduced phase-locking, as is often assumed to take place in schizophrenia patients, may have resulted in a lack of correlation between the two measures as only signal power was increased without influencing phase locking. This indicates less phase spectra reorganization of the ongoing activity. To illustrate this hypothesis, the relationship between mean amplitude and VI was simulated for 2 healthy controls and 2 schizophrenia patients, including perfectly phase-locked responses across trials in healthy controls and poor inter-trial phase locking of responses in patients As shown in Fig. 7, the relationship between higher mean amplitude and lower VI remained evident in healthy controls, similar to the results. Moreover, the lower mean amplitude and VI of subject 1 compared to subject 2 led to a positive correlation between mean amplitude and VI, which also corresponds with the results. In contrast, the equally low mean amplitude in both patients, despite a lower VI in patient 1 than in patient 2 , resulted in a correlation coefficient of mean amplitude and VI around zero. This suggests an influence of the inter-trial phase locking to MMNm and VI, whereas MMNm and VI remain independent of each other.

The limitations of the present study must also be noted: the combination of deviants may limit the conclusions of the present results. However, the similarity of results for the different deviants, the similarity of correlation coefficients between VI and MMNm and the fact that other studies combining different deviants within one design confirmed MMN group differences with only subtle differential effects suggests that the present design did not substantially affect the pattern of results. Gender differences have to be considered in the analysis of VI, as signal variability has been 
Healthy Subject 1

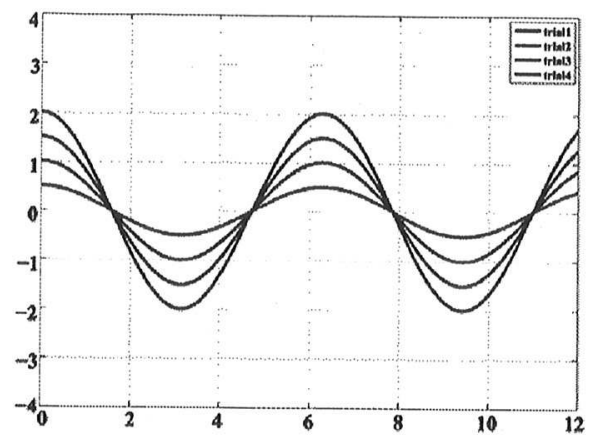

Healthy Subject 2

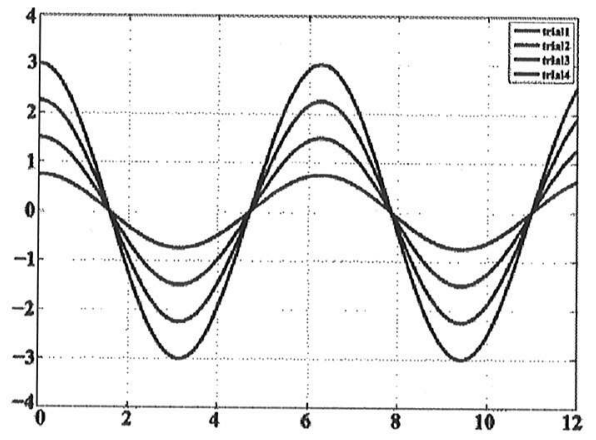

Averages for $\mathrm{HC} 1$ and $\mathrm{SZ} 1$

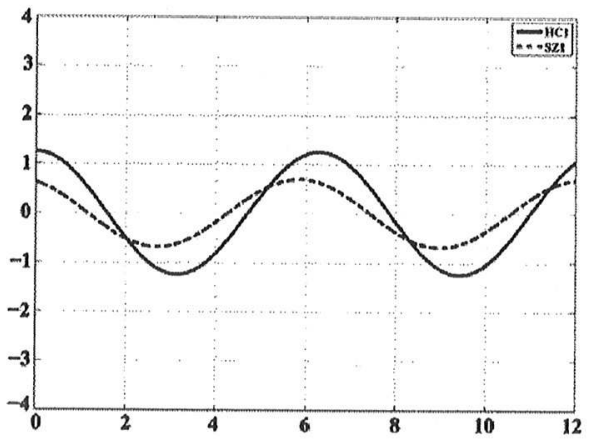

Variability index for HC1 and SZ 1

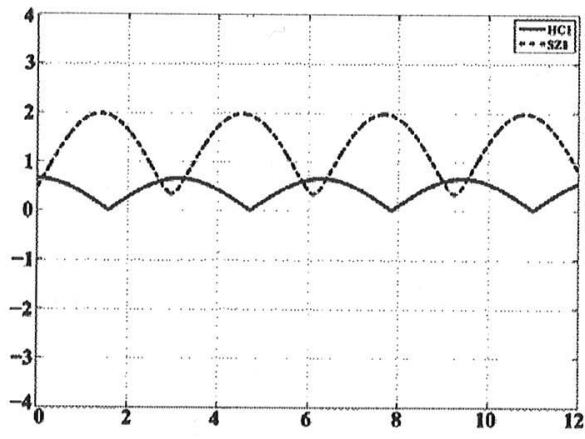

Schizophrenia Patient 1

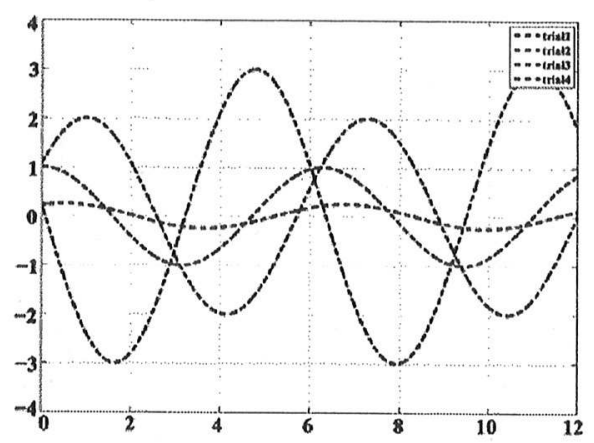

Schizophrenia Patient 2

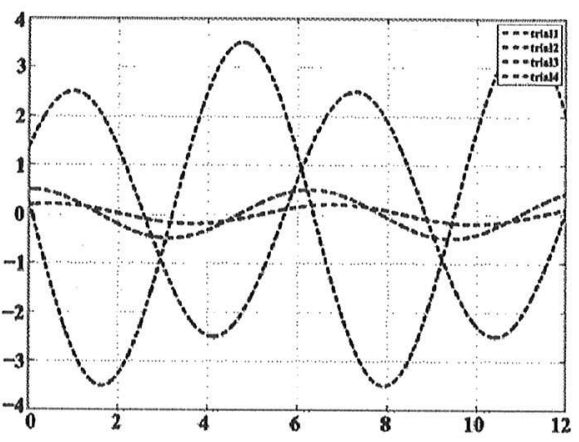

Averages for $\mathrm{HC} 2$ and $\mathrm{SZ} 2$

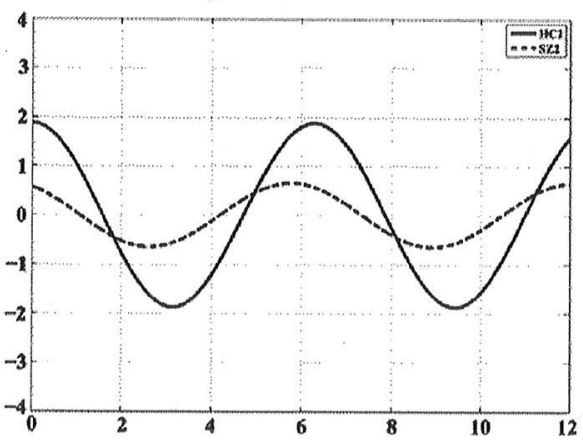

Variability index for HC 2 and SZ 2

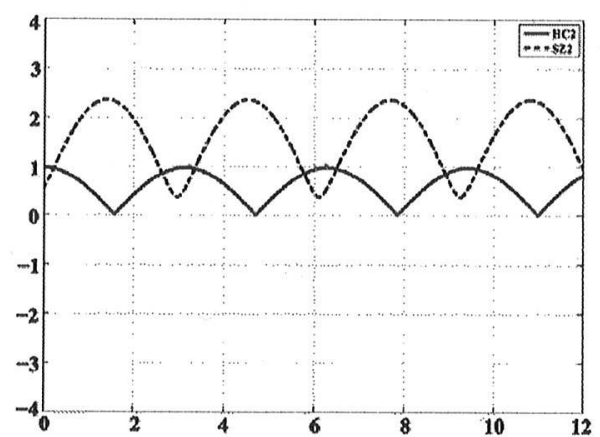

Fig. 7. Modeling the interaction of MMNm amplitude and variability index (VI) by simulation for two healthy subjects (top left, row 1 and 2 ) and two schizophrenia patients (top right, row 1 and 2). Simulation was accomplished with cosine curves. The healthy controls show perfect inter-trial phase locking and the schizophrenia patients show very poor inter-trial phase locking. As a consequence, mean amplitude is larger in healthy controls than in patients, while VI is more pronounced in patients than in healthy controls. Simulation further shows that the VI for HC2 is higher than VI in HC1 and the mean amplitude in HC2 is higher than the amplitude in HC1, which results in a positive correlation of amplitude and VI. For schizophrenia patients, the higher VI did not produce a change in the mean amplitude, and no correlation between amplitude and VI became evident. 
reported to be lower in females than in males (Winterer et al., 2004). The asymmetric distribution of gender in the two samples (one female patient out of 20 , seven female subjects out of 18 in the control group) prevented adequate control for a gender effect. A tendency towards differences in VI between male and female controls is in line with reported gender differences. However, if group differences in VI were mainly due to gender differences, lower VI in females should have reduced the VI in the control group. Since group differences were significant after excluding female subjects (one patient but seven controls) we may assume that they were substantially explained by the difference between schizophrenia patients and controls. Clinical variables that may influence MMN in schizophrenia could not be fully controlled because of the small and selected (medication routine) sample. Although present results did not show a relationship between clinical variables and VI, this should be validated in larger samples, as the duration of an illness has been found to vary with MMN reduction (Näätänen and Kähkönen, 2009) and with the correlation between MMN and oscillatory activity (delta power in QEEG, Kirino, 2007).

In sum, smaller mean MMNm and augmented VI as a consequence of higher response variability across time constitute features related to schizophrenia. Both features seem independent, so that reduced mean MMNm amplitudes in schizophrenia patients cannot be explained primarily as a consequence of increased response variability. Instead, augmented VI and reduced MMNm may both reflect dysfunctions at a very early processing stage in schizophrenia. Augmented VI potentially reflects a greater variability in the neural activation of representations related to one and the same physical stimulus. This could be due to generally diminished 'neurodynamical behavior of prefrontal cortex networks' (Rolls et al., 2008 p. 696) or a weaker representational engram.

\section{Acknowledgment}

This research was supported by the Deutsche Forschungsgemeinschaft (Ro 805/14). All authors declare that they have no actual or potential conflict of interest, including any financial, personal or other relationships with people or organizations, who could inappropriately influence, or be perceived to influence, the presented work.

\section{References}

Ackenheil M, Stotz-Ingenlath G, Dietz-Bauer R, Vossen A. M.I.N.I. Mini International Neuropsychiatric Interview, German Version 5.0.0. München: Psychiatrische Universitätsklinik München 1999.

American Psychiatric Association. Diagnostic and statistical manual of mental disorders (text revision). 4th ed. Washington, DC: American Psychiatric Association: 2000.

Bastiaansen MC, Knösche TR. Tangential derivative mapping of axial MEG applied to event-related desynchronization research. Clin Neurophysiol 2000;111:1300-5.

Beck AT, Steer RA, Brown GK. BDI-II: beck depression inventory-II. San Antonio (TX): Psychological Corp; 1996.

Callaway III E, Jones RT, Donchin E. Auditory evoked potential variability in schizophrenia. Electroenceph Clin Neurophysiol 1970;29:421-8.

Deacon D, Nousak J, Pilotti M, Ritter W, Yang CM. Automatic change detection: does the auditory system use representations of individual stimulus features or gestalts? Psychophysiology 1998;35:413-9.

Deouell LY, Bentin S. Variable cerebral responses to equally distinct deviance in four auditory dimensions: a mismatch negativity study. Psychophysiology 1998:35:745-54.

Elbert T, Ray WJ, Kowalik ZBJ, Skinner JE, Graf KE, Birbaumer N. Chaos and physiology: deterministic chaos in excitable cell assemblies. Physiol Rev $1994 ; 74: 1-47$.

Fehr T, Kissler J, Wienbruch C, Moratti S, Rockstroh B, Elbert T. Source distribution of neuromagnetic focal slow waves and MEG-delta activity in schizophrenic patients. Biol Psychiatry 2001:50(2):108-16.

Fehr T, Kissler J, Wienbruch C, Moratti S, Elbert T, Watzl H, et al. Source distribution of neuromagnetic slow wave activity in schizophrenic patients - effects of activation. Schizophr Res 2003:63(1-2):63.

Fisher DJ, Grant B, Smith DM, Knott VJ. Effects of deviant probability on the 'optimal' multi-feature mismatch negativity (MMN) paradigm. Int J Psychophysiol 2011;79:311-5.
Garrido Ml, Fristin KJ, Kiebel SJ, Stephan KE, Baldeweg T, Kilner JM. The functional anatomy of the MMN: a DCM study of the roving paradigm. Neuroimage 2008:42:936-44

Garrido MI, Kilner JM, Stephan KE, Friston KJ. The mismatch negativity: a review of underlying mechanisms. Clin Neurophysiol 2009;120:453-63.

Hämäläinen $M$, Hari R, Ilmoniemi RJ, Knuutila J, Lounasmaa oV, Magnetoencephalography - theory, instrumentation, and applications to noninvasive studies of the working human brain. Rev Mod Phys 1993:65:413-97.

Hedges LV, Olkin I. Statistical methods for meta-analysis. London: Academic Press: 1985.

Horton J. Millar A, Labelle A, Knott VJ. MMN responsivity to manipulations of frequency and duration deviants in chronic, clozapine-treated schizophrenia patients. Schizophr Res 2010; (Epub ahead of print).

lyer D, Zouridakis G. Single-trial analysis of the auditory N100 improves separation of normal and schizophrenia subjects. Conf Proc IEEE Eng Med Biol Soc 2008:3840-3.

Jansen BH, Hu L, Boutros NN. Auditory evoked potential variability in healthy and schizophrenia subjects. Clin Neurophysiol 2010;121(8):1233-9.

Kircher TT, Rapp A, Grodd W, Buchkremer G, Weiskopf N, Lutzenberger W, et al. Mismatch negativity responses in schizophrenia: a combined fMRI and wholeMead MEG study. Am J Psychiatry 2004;161:294-304.

Kirino E. Mismatch negativity correlates with delta and theta EEG power in schizophrenia. Int J Neurosci 2007;117:1257-79.

Kissler J. Müller MM, Fehr T, Rockstroh B, Elbert T. MEG gamma band activity in schizophrenia patients and healthy subjects in a mental arithmetic test and at rest. Clin Neurophysiol 2000;111:2079-87.

Kreitschmann-Andermahr I, Rosburg T, Meier T, Volz HP, Nowak H, Sauer H. Impaired sensory processing in male patients with schizophrenia: a magnetoencephalographic study of auditory mismatch detection. Schizophr Res 1999;35:121-9.

Leicht G, Kirsch V, Giegling I, Karch S, Hantschk I, Möller HJ, et al. Reduced early auditory evoked gamma-band response in patients with schizophrenia. Biol Psychiatry 2010;67:224-31.

Lukoff D, Nuechterlein KH, Ventura J. Manual for the expanded Brief Psychiatric Rating Scale (BPRS). Schizophr Bull 1986:12:196-204.

Magno E, Yeap S, Thakore JH, Garavan H, De Sanctis P, Foxe JJ. Are auditory-evoked frequency and duration mismatch negativity deficits endophenotypic for schizophrenia? High-density electrical mapping in clinically unaffected firstdegree relatives and first-episode and chronic schizophrenia. Biol Psychiatry 2008;64:385-91.

Maris E, Oostenveld R. Nonparametric statistical testing of EEG- and MEG-data. J Neurosci Methods 2007;164:177-90.

Matsubayashi J, Kawakubo Y, Suga M, Takei Y, Kumano S, Fukuda M, Itoh K, Yumoto $M$, Kasai $K$. The influence of gender and personality traits on individual difference in auditory mismatch: a magnetoencephalographic (MMNm) study. Brain Res 2008;1236:159-65.

Michie PT. What has MMN revealed about the auditory system in schizophrenia? Int J Psychophysiol 2001:42:177-94.

Michie PT, Budd TW, Todd J, Rock D, Wichmann H, Box J. Jablensky AV. Duration and frequency mismatch negativity in schizophrenia. Clin Neurophysiol 2000;111:1054-65.

Möcks J, Gasser T, Köhler W. Basic statistical parameters of event-related potentials. J Psychophysiol 1988:2:61-70

Näätänen R. Attention and brain function. Hillsdale, $\mathrm{NJ}$ : Lawrence Erlbaum Associates; 1992.

Näätänen R, Kähkönen S. Central auditory dysfunction in schizophrenia as revealed by the mismatch negativity (MMN) and its magnetic equivalent MMNm: a review. Int J Neuropsychoph 2009;12:125-35.

Näätänen R, Paavilainen P, Rinne T, Alho K. The mismatch negativity (MMN) in basic research of central auditory processing: a review. Clin Neurophysio] 2007:118:2544-90.

Näätänen R, Pakarinen S, Rinne T, Takegata R. The mismatch negativity (MMN) towards the optimal paradigm. Clin Neurophysiol 2004;115:140-4.

Pakarinen S, Houtilainen M, Näätänen R. The mismatch negativity (MMN) with no standard stimulus. Clin Neurophysiol 2010;121:103-1050.

Pekkonen E, Katila H, Ahveninen J, Karhu J, Houtilainen M, Tihonen J. Impaired temporal lobe processing of preattentive auditory discrimination in schizophrenia. Schizophr Bull 2002;28:467-74.

Petermann M, Kummer P, Burger $M$, Lohscheller J, Eysholdt U, Döllinger $M$ Statistical detection and analysis of mismatch negativity derived by a multideviant design from normal hearing children. Hear Res 2009;347:128-36.

Popov T, Jordanov T, Weisz N, Elbert T, Rockstroh B, Miller GA. Evoked and induced oscillatory activity contributes to abnormal auditory sensory gating in schizophrenia. Neuroimage 2011;56(1):307-14

Reite M, Teale P, Rojas DC. Magnetoencephalography: applications in psychiatry. Biol Psychiatry 1999;45:1553-63.

Rinne T, Alho K, Ilmoniemi RJ, Virtanen J, Näätänen R. Separate time behaviors of the temporal and frontal mismatch negativity sources. Neuroimage 2000;12:14-9.

Rockstroh BS, Wienbruch C, Ray WJ, Elbert T. Abnormal oscillatory brain dynamics in schizophrenia: a sign of deviant communication in neural network? BMC Psychiatry 2007;7:44.

Rolls ET, Loh M, Deco G, Winterer G. Computational models of schizophrenia and dopamine modulation in the prefrontal cortex. Nat Rev Neurosc 2008:9:696-709. 
Rosburg T, Kreitschmann-Andermahr L, Sauer H. Mismatch negativity in schizophrenia research. An indicator of early processing disorders of acoustic information. Nervenarzt 2004;75(7):633-41.

Roth A, Roesch-Ely D, Bender S, Weisbrod M, Kaiser S. Increased event-related potential latency and amplitude variability in schizophrenia detected through wavelet-based single trial analysis. Int J Psychophysiol 2007;66:244-54.

Siekmeier PJ. Stufflebeam SM. Patterns of spontaneous magnetoencephalographic activity in patients with schizophrenia. J Clin Neurophysiol 2010;27:179-90.

Tallon-Baudry C, Kreiter A, Bertrand $O$. Sustained and transient oscillatory responses in the gamma and beta bands in a visual short-term memory task in humans. Visual Neuroscience 1999;16:449-59.

Tervaniemi M, Sinkkonen J, Virtanen J, Kallio J, Ilmoniemi RJ, Salonen O, et al. Testretest stability of the magnetic mismatch response (MMNm). Clin Neurophysiol 2005:116:1897-905

Thönnessen H, Zvyagintsev M, Harke KC, Boers F, Dammers J, Norra $\mathrm{Ch}$, et al Optimized mismatch negativity paradigm reflects deficits in schizophrenia patients. A combined EEG and MEG study. Biol Psychol 2008;77:205-16.

Todd J, Michie PT, Schall U, Karayanidis F, Yabe H, Näätänen R. Deviant matters: duration, frequency, and intensity deviants reveal different patterns of mismatch negativity reduction in early and late schizophrenia. Biol Psychiatry 2008:63:58-64.

Turetsky Bl, Moberg PJ. An odor-specific threshold deficit implicates abnormal intracellular cyclic AMP signaling in schizophrenia. Am J Psychiatry 2009:166:226-33.

Umbricht $D$, Krljes S. Mismatch negativity in schizophrenia: a meta-analysis. Schizophr Res 2005;76:1-23.

Winterer G, Ziller M, Dorn H, Frick K, Mulert C, Wuebben Y, et al. Schizophrenia: reduced signal-to-noise ratio and impaired phase-locking during information processing. Clin Neurophysiol 2000;111:837-49.

Winterer G, Coppola R, Goldberg TE, Egan MF, Jones DW, Sanchez CE, et al. Prefiontal broadband noise, working memory, and genetic risk for schizophrenia. Am J Psychiatry 2004:161:490-500.

Winterer G, Egan M, Kolachana B, Goldberg T, Coppola R, Weinberger D. Prefrontal electrophysiologic "noise" and catechol-0-methyltransferase genotype in schizophrenia. Biol Psychiatry 2006;60:578-84.

Yabe H, Sutoh T, Matsuoka T, Asai R, Hiruma T, Sato $Y$, et al. Transient gamma-band response is dissociated from sensory memory as reflected by MMN. Neurosci 